\title{
Endophilin Is Critically Required for Synapse Formation and Function in Drosophila melanogaster
}

\author{
Richa Rikhy, ${ }^{*}$ Vimlesh Kumar, ${ }^{*}$ Rohit Mittal, and K. S. Krishnan \\ Tata Institute of Fundamental Research, Mumbai 400005 India
}

Studies in cell-free systems and the lamprey giant synapse have implicated crucial roles for amphiphysin and endophilin in synaptic transmission. However, null mutants at the amphiphysin locus of Drosophila are viable and have no demonstrable synaptic vesicle-recycling defect. This has necessitated a reexamination of the role of Src homology 3 domain-containing proteins in synaptic vesicle recycling. In this report, we show that endophilin-deficient eye clones in Drosophila have an altered electroretinogram. A characteristic of this defect is its

Recruitment of a whole host of components whose activities facilitate endocytic fission by dynamin is an attractive alternative to a purely mechanochemical role for this molecule (Takei et al., 1998; Simpson et al., 1999; Stowell et al., 1999; Marks et al., 2001). Initially identified in biochemical studies, Src homology 3 (SH3) domain-containing proteins such as amphiphysin and endophilin have been implicated in the endocytic retrieval of vesicular components in association with dynamin (Shupliakov et al., 1997; Simpson et al., 1999). Much of the evidence has come from cell-free systems and the lamprey giant synapse, demonstrating the role of endophilin and amphiphysin in synaptic function (Takei et al., 1999; Farsad et al., 2001). However, knock-out of the amphiphysin locus in Drosophila yielded viable flies without any neuronal defect, which calls into question the importance of an amphiphysin-like molecule in synaptic vesicle recycling (Leventis et al., 2001; Razzaq et al., 2001; Zelhof et al., 2001). endophilin, like amphiphysin, has been shown to bind dynamin by its $\mathrm{SH} 3$ domain in vitro, and it has an appropriate lipid-modifying activity, which might prove useful in inducing membrane curvature (David et al., 1996; Schmidt et al., 1999; Simpson et al., 1999). The only in vivo study in the lamprey synapse used injection of specific antibodies and antimorphic peptides to show an involvement of the protein in vesicle recycling (Ringstad et al., 1999; Gad et al., 2000). However, an in vitro study suggests that endophilin may have a role in stabilizing dynamin-coated tubules, thereby inhibiting the vesiculation process (Farsad et al., 2001). The facility of Drosophila genetics has allowed us to re-examine and further

Received Feb. 20, 2002; revised June 28, 2002; accepted July 3, 2002.

This work was supported by grants from the Department of Biotechnology and Department of Science and Technology (K.S.K.), in addition to funds from the Tata Institute of Fundamental Research. We thank Veronica Rodrigues for her constructive suggestions regarding this manuscript, Erich Buchner for anti-CSP antibodies, Mani Ramaswami for rabbit anti-dynamin antibodies, and Ram Kumar for rat anti-dynamin antibodies.

*R.R. and V.K. contributed equally to this work.

Correspondence should be addressed to Dr. K. S. Krishnan, Tata Institute of Fundamental Research, Homi Bhabha Road Colaba, Mumbai 400005 India. E-mail: ksk@tifr.res.in.

Copyright (C) 2002 Society for Neuroscience $0270-6474 / 02 / 227478-07 \$ 15.00 / 0$ aggravation during heightened visual stimulation. It is shown that endophilin is primarily required in the nervous system. Decreased endophilin activity results in alterations in the neuromuscular junction structure and physiology. Immunofluorescence studies show colocalization of endophilin with dynamin consistent with a possible role in synaptic vesicle recycling.

Key words: Drosophila melanogaster; endophilin; transposoninduced mutant; hypomorph; electroretinogram; neuromuscular junction

address the in vivo neuronal requirement and function of endophilin. Here we report neural phenotypes of Drosophila endophilin mutants. Although the more severe mutant allele is lethal and affected in synaptic transmission in mutant clones in the eye, the formation and function of synapses at the larval neuromuscular junction (NMJ) are shown to be affected in the hypomorph.

\section{MATERIALS AND METHODS}

Drosophila stocks. EP0927, an insertion in the endophilin gene, was obtained from Exelixis Inc. (San Francisco, CA). An EP0927 FRT 82B stock was generated by meiotic recombination with P (neo FRT 82B) selected by growing on gentamicin. An Flp;GMR-Hid FRT 82B stock was generated by standard genetics from Ey Gal4 UAS Flp;GMR-Hid FRT 82B and Ey Flp were obtained from the Bloomington Stock Center (Bloomington, IN) and Iswar Hariharan (Massachusetts General Hospital, Boston, MA), respectively. The Myc-tagged glutamate receptor (GluR) line GluR2a was obtained from Aaron DiAntonio (Washington University, St. Louis, MO). Armadillo (Arm) Gal4 was obtained from J. P. Vincent (National Institute for Medical Research, Cambridge, UK). All other standard fly stocks were part of the Tata Institute of Fundamental Research Stock Collection. Arm Gal4;EP0927/TM6b was maintained as a stock, and wandering non-tubby third instar larvae were picked for the experiments. The flies were reared at $25^{\circ} \mathrm{C}$ in standard sugar/agar medium with yeast in glass vials and bottles.

Cloning the endophilin ORF and semiquantitative PCR. Gene-specific primers (5'-GACAGCGGCCGCCATGGCTTTCGCCGG-3' and 5'ATTGGTACCCTAGAAGAACTCAGGTGG-3') designed based on the Drosophila genome sequence were used to amplify the predicted endophilin ORF by reverse transcription-PCR (RT-PCR) from RNA extracted from the head of the adult fly. For semiquantitative RT-PCR, $2 \mu \mathrm{g}$ of RNA from the heads of wild type (Canton Special) or EP0927/EP0927 clones was reverse-transcribed. For pilot PCR experiments, $0.5 \mu \mathrm{l}$ of cDNA per $50 \mu \mathrm{l}$ reaction volume was used to determine the linear range with respect to the number of cycles. Twenty-eight cycles of PCR were performed for endophilin and the internal control gene, $r p 49$. The primer pairs used were $5^{\prime}$ -

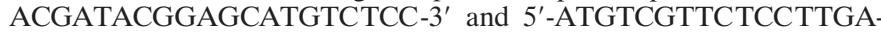
AGGC-3' for endophilin and 5'-AGATCGTGAAGAAGCGCACC-3' and 5'-CGATCCGTAACCGATGTTGG-3' for $r p 49$.

Phototaxis. A countercurrent apparatus described by Benzer (1967) was used to measure phototaxis. The number of flies in the lower block was estimated at the end of the experiment, and the phototaxis index (PhI) was calculated as follows: $\mathrm{PhI}={ }^{\mathrm{r}} \Sigma_{0} n_{\mathrm{i}} t_{\mathrm{i}} / r \times{ }^{\mathrm{r}} \Sigma_{0} n_{\mathrm{i}}$, where $r$ equals the total number of tubes on one side, $\mathrm{n}_{\mathrm{i}}$ equals the number of flies in the $i$ th tube, and $t_{\mathrm{i}}$ equals tube number. 
Antibody generation and Western blotting. Two primers (5'-GCAGGATCCATGGCTTTCGC-3' and 5'-TCGAAAAGCTTGTCGTCGACACGCG-3') were used to amplify $\sim 1.03 \mathrm{~kb}$ of the $5^{\prime}$ end of the endophilin ORF, including the enzymatic and coiled-coil domains (Endo $\Delta \mathrm{SH} 3$ ). The PCR product was cloned in frame with glutathione $S$-transferase (GST) in the pGEX-KG vector and transformed into Escherichia coli XL-1 blue. The expression of the fusion protein and subsequent purification on a glutathione agarose column were done using standard methods. Endo $\Delta \mathrm{SH} 3$ was injected into rabbits to raise polyclonal antibodies. The antiserum was depleted of anti-GST antibodies by passing it over glutathione agarose beads charged with bacterially expressed GST. Rat anti-dynamin antibody was similarly generated by injecting bacterially expressed and purified Drosophila dynamin in Sprague Dawley rats (R. Mittal, personal communication). Western blotting was performed with purified proteins and Drosophila homogenates to ascertain specificity (data not shown).

Immunostaining. Wandering third instar larvae were pinned down dorsally on a Sylgard dish and dissected in cold calcium-free HL3 saline (in mM: $70 \mathrm{NaCl}, 5 \mathrm{KCl}, 20 \mathrm{MgCl}_{2}, 10 \mathrm{NaHCO}_{3}, 5$ trehalose, 115 sucrose, and 5 HEPES, pH 7.3) to expose the neuromuscular junction. Samples were fixed in $3.5 \%$ paraformaldehyde in PBS, washed in PBS containing $0.15 \%$ Triton $\mathrm{X}-100$, and incubated overnight at $4^{\circ} \mathrm{C}$ with the primary antibody. Fluorophore-coupled secondary antibodies were used to visualize antibody binding on a Bio-Rad Radiance (Hercules, CA) 2000 confocal microscope, and images were acquired on a Laser Sharp 2000 (Bio-Rad, Hercules, CA) and processed with Adobe Photoshop 5.5 (Adobe Systems, San Jose, CA) and Metamorph (Universal Imaging, Downing, PA).

Electroretinogram recordings. Extracellular recordings of light-evoked visual responses were made from eyes of 2- to 3-d-old flies grown at $25^{\circ} \mathrm{C}$. Flies, lightly immobilized by cooling on ice, were mounted upright on modeling clay with the right eye facing the light. Recording electrodes filled with $3 \mathrm{M} \mathrm{KCl}$ (tip resistance of 3-5 $\mathrm{M} \Omega$ ) were placed in contact with the eye and a reference electrode was inserted into the thorax. Light pulses of the duration indicated were delivered from an optical fiber output placed 3-4 cm from the eye of the fixed fly. Signals were amplified using an intracellular preamplifier (IX2-700 Dual Intracellular Preamplifier; Dagan, Minneapolis, MN), and data were acquired directly from the oscilloscope (Tektronix, Wilsonville, OR) connected to a computer.

Neuromuscular junction recordings. Wandering third instar larvae were dissected in calcium-free HL3 saline; a $1.5 \mathrm{~mm}$ concentration of $\mathrm{CaCl}_{2}$ was added for recordings. The same saline was used in the suction electrode to stimulate the nerve-innervating segment $\mathrm{A} 2$. The recording electrode, with a resistance of 20-30 M , was inserted in muscle 6 of segment A2. For evoked junction potential (EJP) measurements, the larvae were stimulated at $3-5 \mathrm{mV}$ at $1 \mathrm{~Hz}$ stimulation. Synaptic depression was performed at $10 \mathrm{~Hz}$ for $5 \mathrm{~min}$. The signal was amplified using Axoclamp 2B acquired by Digidata 1320 on pClamp8 software from Axon Instruments (Foster City, CA); for stimulation, an S48 Grass stimulator and isolator (Grass Instruments, West Warwick, RI) was used. miniature EJP (MEJP) amplitude is a mean from events observed over $25 \mathrm{sec}$ in each larva. The EJP amplitude is a mean from events recorded for $60 \mathrm{sec}$. Quantal content is calculated as the ratio of the mean of the EJP and MEJP amplitude. The MEJPs were extracted, and averages were computed using Mini Analysis software (Synaptosoft Inc., Decatur, GA).

Assay for vesicle recycling. FM1-43 dye uptake studies were performed according to protocols reported by Ramaswami et al. (1994) with a 5 min fixation in $3.5 \%$ paraformaldehyde in PBS after uptake. We found that this procedure made the preparations stable and easier to image without recourse to a water lens and did not affect the results in any way. Images were captured using a Zeiss (Oberkochen, Germany) Axiovert microscope connected to a Hamamatsu (Shizouka, Japan) cooled CCD camera acquired with Zeiss Axiovision.

\section{RESULTS}

\section{EP0927 is a P insertion in the $5^{\prime}$-untranslated region of} Drosophila endophilin

A putative endophilin was identified in the completed Drosophila genome sequence on the basis of its identity with the mammalian protein (45-47\%) (Myers et al., 2000). There is only one such gene in Drosophila, compared with three in vertebrates. The vertebrate genes endophilin I, endophilin II, and endophilin III show a differential tissue expression (Ringstad et al., 1997). The protein sequence shows homologous domain arrangement with a putative lysophosphatidic acid acyl transferase domain toward the $\mathrm{N}$ terminal, a middle coiled-coil domain, and a C-terminal SH3binding domain. We confirmed the expression of this gene by using specific primers (see Materials and Methods) made according to the available sequence and amplifying the cDNA by RTPCR from the head of an adult fly mRNA (data not shown). A continuous $1.11 \mathrm{~kb}$ (intronless) exon encodes Drosophila-specific endophilin. Conceptual translation of this gene gives rise to a 369 aa protein. The molecular identification of P-element insertion positions has made gene-specific knock-outs a useful possibility. One such P-element-containing line, EP0927, from the Rorth collection, was found to be situated in the $5^{\prime}$ untranslated region of this gene according to Berkeley Drosophila genome project information (Rorth, 1996). EP0927 is homozygous lethal at the second instar larval stage. We verified that the $\mathrm{P}$ element was inserted $431 \mathrm{bp}$ upstream of the predicted translation start site by rescue of the genome region flanking the $\mathrm{P}$ element. This line has appropriate UAS elements in the correct orientation to allow expression of endophilin in a tissue-specific manner by using different Gal4 drivers. Expression of this gene using a panneuronal Ga14 line Elav C155-Gal4 resulted in completely viable flies, suggesting a neuronal requirement for the gene. Therefore, we could use this line effectively as an endophilin loss-of-function mutant to address its role in synaptic transmission using genetics, immunostaining, and electrophysiology.

\section{Eye clones carrying homozygous EP0927 have electroretinographic defects}

To look for functional defects in cells lacking endophilin, we generated eye clones homozygous for the endophilin mutation. This was achieved by crossing EP0927 FRT 82B to Ey Flp;GMRHid FRT 82B (mutant clones) (Stowers and Schwarz, 1999). Eye clones generated by crossing to Ey Gal4 UAS Flp would in principle be able to drive the expression of endophilin in otherwise mutant cells (rescued clones) and served as controls for the experimental Ey Flp-generated clones. The clonal tissue covered the entire eye, because the rest of the tissue would be killed by hid expression under the GMR promoter. Experimental (Ey Flp) and control (Ey Gal4 UAS Flp) clones were morphologically indistinguishable (Fig. 1A). However, there was a distinct difference in electroretinogram (ERG) recordings.

An ERG consists of fast on and off transients when the light is switched on and off, respectively, and a slow depolarization. The slow depolarization is primarily a response of photoreceptor cells to light. The on and the off transients are indicative of synaptic transmission from the photoreceptor cells to the laminar neurons (Heisenberg, 1971). In a large number of flies containing the mutant clone, we observed a constitutive loss of on and off transients. However, when mutant flies showing the transients were examined on the first day after eclosion, the on transient was present in all cases and the off transient was present in some. In both flies containing the mutant and the rescued eye clones, the on transient was intact in the first few flashes of light given at $2 \mathrm{~Hz}$ (Fig. 1Bi). However, after heightened photostimulation, the transient remained normal for the wild-type and rescued clones and disappeared in an activity-dependent manner for the endophilin mutant clones (Fig. 1Bii,Biii). These transients reappear after less than a $15 \mathrm{sec}$ rest period in the dark (Fig. 1Biv). The flies with mutant clones are compromised in their ability to photo tax (move toward light) (Fig. 1C). The heads of these flies show a lowered level of endophilin transcript (Fig. 1D). This phenotype 
A

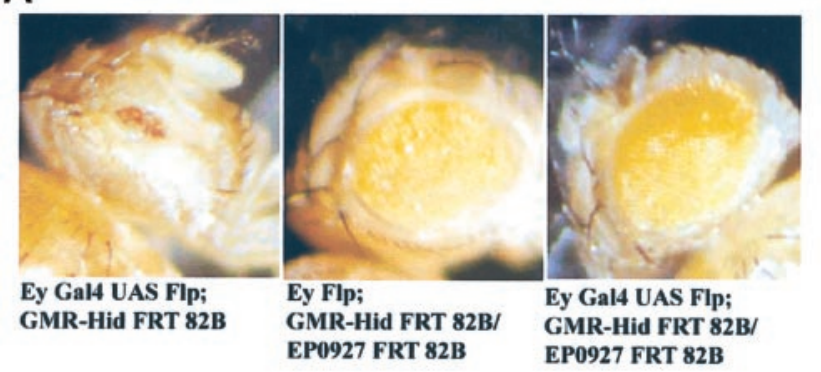

B
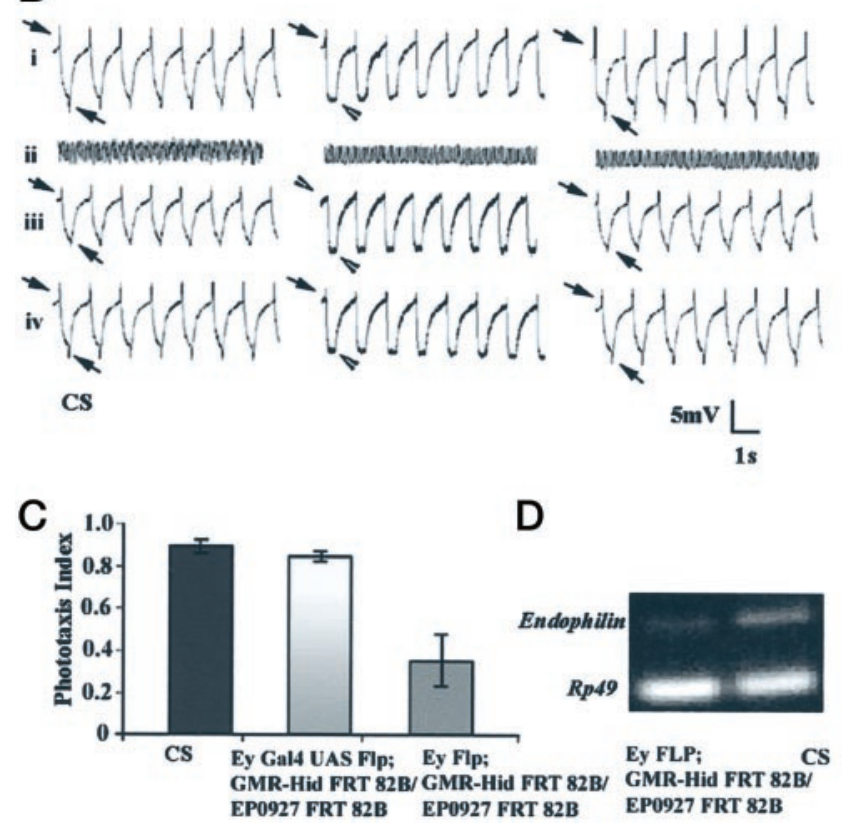

Figure 1. A P-element-mediated endophilin mutant shows an activitydependent loss in synaptic transmission in the eye. A, Drosophila eye bright-field images depicting the original eyeless stock followed by the rescued eye clones (Ey Gal4 UAS Flp;GMR-Hid FRT 82B/EP0927 FRT 82B) and the mutant eye clones (Ey Flp;GMR-Hid FRT 82B/EP0927 FRT 82B) (see Results for details). $B$, ERG recordings from wild-type, rescued, and mutant clones. Stimulation with a light pulse at $2 \mathrm{~Hz}(i)$ followed by a heightened $10 \mathrm{~Hz}$ stimulation for $1 \mathrm{~min}$ (ii) and immediate ERG recording (iii) is shown. Finally, flies were dark-adapted for $15 \mathrm{sec}$, and the ERG was recorded (iv). Solid arrows point to transients and open arrowheads point to a loss of transients. $C$, Phototaxis for wild-type flies with endophilin-rescued clones and mutant clones, respectively $(n=3$ in each case). The $\mathrm{PhI}$ is indicated on the $y$-axis. Error bars indicate SD. $d$, Semiquantitative RT-PCR for endophilin transcript levels from the heads of adult wild-type and mutant eye clones. CS, Canton Special; Rp49, concentration control for mRNA.

is in agreement with the possibility that the first few flashes of light deplete terminals of vesicles because of compromised vesicle recycling and that synaptic transmission is disrupted.

\section{Endophilin and dynamin localize in similar zones in presynaptic boutons}

We generated polyclonal antibodies in rabbits to a GST fusion of the $\mathrm{N}$ terminal of endophilin (GST-Endo $\Delta \mathrm{SH} 3$ ) from 1 to 340 aa ( $\sim 37 \mathrm{kDa})$ (Fig. $2 A$, schematic). We show that the antibodies generated are specific for the original $37 \mathrm{kDa}$ pure protein and recognize a single protein in the fly head extract at an expected 41 kDa (Fig. 2A). Anti-endophilin strongly stains the synapse at the neuromuscular junction (Fig. $2 B$ ). This staining is not detected
A
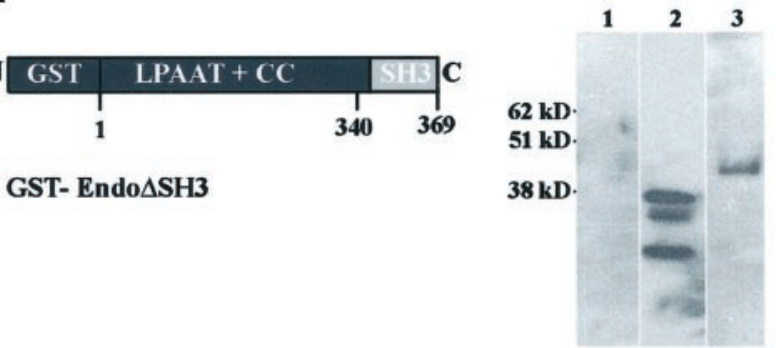

B
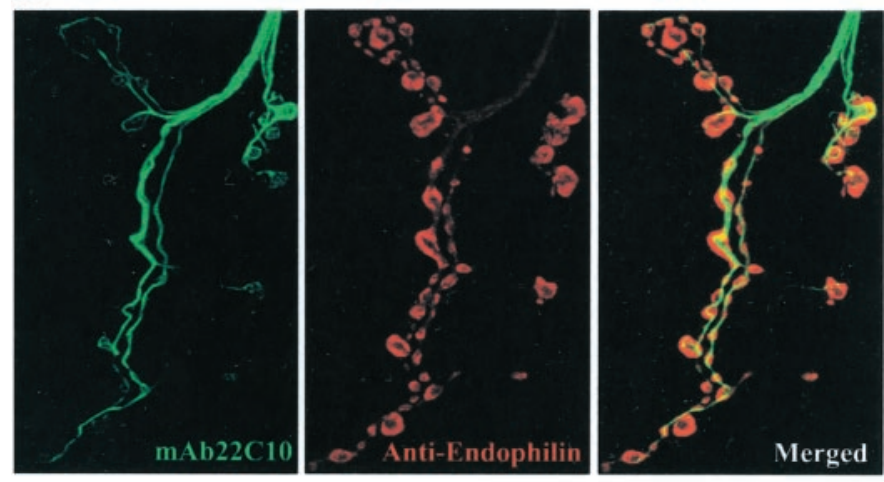

C

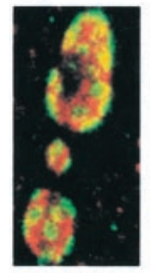

GluR11a Dynamin

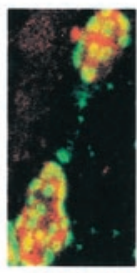

GluR11a Endophilin
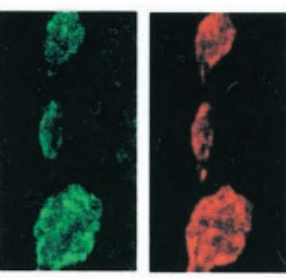

Dynamin Endophilin

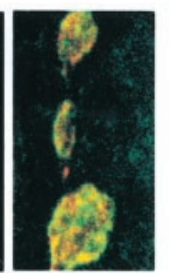

Dynamin Endophilin

Figure 2. Characterization of the anti-endophilin antibody. A, The schematic shows the region of the endophilin ORF (GST-Endo $\Delta \mathrm{SH} 3$ ) to which the rabbit polyclonal antibody is raised. At right is a Western blot assaying the specificity of the antibody obtained. The preimmune serum shows an absence of any staining against fly head extract (1). GSTEndo $\Delta \mathrm{SH} 3$ is cleaved to release GST and Endo $\Delta \mathrm{SH} 3$. The immune serum $(1: 10,000)$ recognizes GST $(27 \mathrm{kDa})$ and the pure protein $(37 \mathrm{kDa})(2)$. The antibody recognizes a single band $(41 \mathrm{kDa})$ from fly head extracts (3). $B$, Anti-endophilin and $\mathrm{mAb} 22 \mathrm{C} 10 /$ anti-Futsch staining at the neuromuscular junction of the third instar larvae. $C$, Left, Double labeling of the motor terminals of larvae from the Myc-tagged glutamate receptor stock with anti-endophilin or anti-dynamin and anti-Myc for the glutamate receptor. Right, Double staining of motor terminals of wild-type larvae with rat anti-dynamin and anti-endophilin.

when treated with preimmune sera and abolished when antibody is titrated with pure protein (data not shown). We noticed that the endophilin antibody highlights synaptic regions, which are excluded from staining by monoclonal antibody (mAb) 22C10/ anti-Futsch. We did a double labeling with dynamin or endophilin and glutamate receptor using anti-Myc antibodies on a transgenic line carrying a Myc-tagged glutamate receptor (Fig. 2C). Glutamate receptor staining would effectively label active zones that are sites for exocytosis at the synapse. We found that both dynamin and endophilin have a complementary staining pattern to that of the glutamate receptor. Endophilin stains endocytic hot spots or peri-active zones in a manner similar to that reported for 
dynamin (Estes et al., 1996; Roos and Kelly, 1999). As expected, there is a considerable overlap of endophilin and dynamin in a different double-labeling experiment, as shown in Figure $2 C$.

\section{Armadillo Gal4-driven EP0927 flies have virtually no detectable endophilin protein}

EP0927 when driven by Elav Gal4 yielded adult flies that were completely viable but showed a sluggish phenotype. When we attempted the same rescue experiments using a ubiquitous driver, Arm Gal4, we unexpectedly obtained flies that progressed well through development but were unable to eclose from the pupal case (Fig. $3 A$ ). It became important at this point to compare the strength of the two drivers in the nervous system by crossing them to UAS-GFP (green fluorescent protein). We found that the Elav Gal4;UAS-GFP combination fluoresces more strongly in the brain of third instar larvae compared with Arm Gal4;UAS-GFP, suggesting that this phenotype may be attributable to a lower expression of the endophilin protein in these neurons (data not shown). We used wandering third instar larvae of Arm Gal4; EP0927/EP0927 to determine whether this was the case. Indeed, we found that there was little detectable endophilin immunoreactivity at the neuromuscular junction in these larvae (Fig. 3B). The staining for synapse markers such as cysteine-string protein (CSP) and dynamin (data not shown) was normal, and the endophilin immunoreactivity was present in Elav Gal4;EP0927/ EP0927. We conclude that Arm Gal4;EP0927/EP0927 is an effective hypomorph for endophilin in the nervous system. Arm Gal4 was used in a recent report (Guichet et al., 2002) to completely rescue a hypomorphic combination of endophilin mutants. We conclude that the Arm Gal4;EP0927/EP0927 combination is weaker than these endophilin mutants, because the level of endophilin is almost undetectable.

\section{Modification of synapse structure resulting from lower levels of endophilin}

After staining Arm;EP0927/EP0927 larval neuromuscular junctions with anti-CSP (Fig. $3 B, C$ ), we found that the synapse is larger and has a greater number of boutons. The number of boutons was $75 \%$ higher than wild type. (Fig. $3 D$ ). The boutons seem morphologically smaller in size compared with wild type. This is shown by double staining with anti-HRP and anti-CSP at the neuromuscular junction (Fig. $3 C$ ). The increase in the number of boutons was rescued in Elav Gal4;EP0927/EP0927, although some boutons did appear smaller compared with wild type. We conclude that the lower levels of endophilin cause synapse sprouting. We ruled out the possibility of this phenotype resulting because of a misexpression of endophilin in muscle that was attributable to a lack of any detectable muscle immunoreactivity or alterations in muscle structure per se. The synapse architecture is unchanged when EP0927 is driven in a muscle-specific manner in Mhc Gal4;EP0927 and in a background control of Arm Gal4;EP0927/TM3.

\section{Changes in synapse function associated with lowered levels of endophilin}

We sought to determine whether the hypomorph of endophilin created by the combination Arm Gal4;EP0927/EP0927 has a defect in synaptic function by electrophysiology. We found that when dissected out of their pupal cases, the escaper flies showed normal ERGs, and the amplitude of their response did not diminish with increased photostimulation (data not shown). The

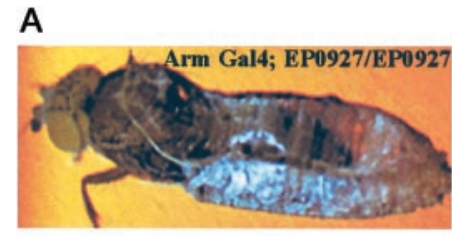

B
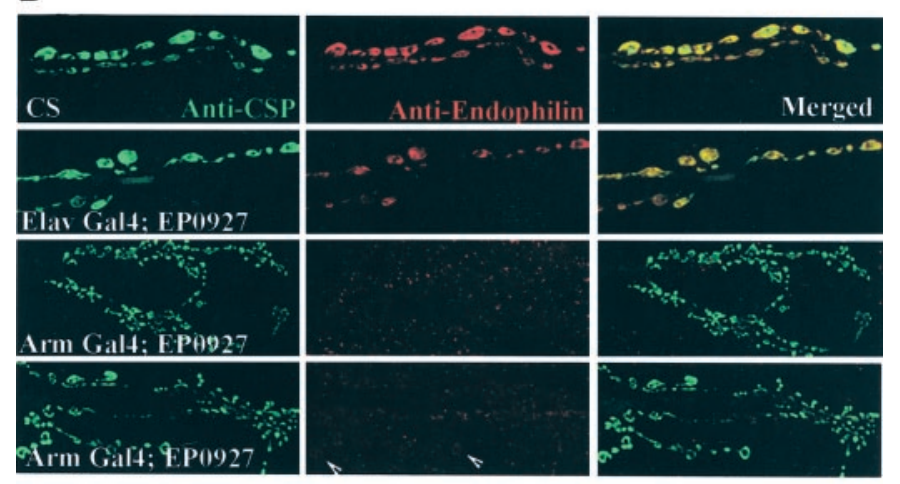

C
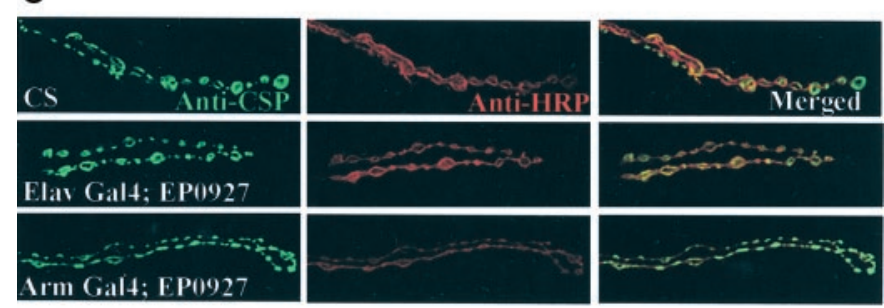

D

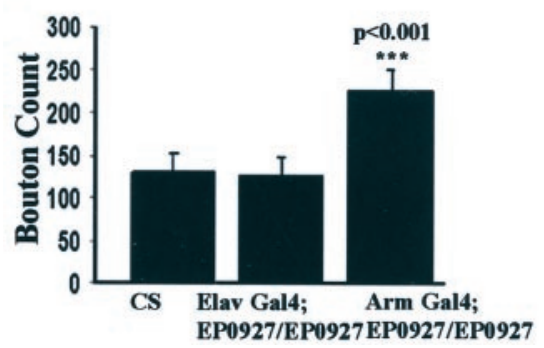

Figure 3. Phenotypes of EP0927 driven by Armadillo Gal4. A, Brightfield image of an adult fly with the genotype Arm Gal4;EP0927/EP0927 unable to eclose from the pupal case. $B$, Anti-CSP and anti-endophilin staining at the neuromuscular junction of wild-type Elav Gal4;EP0927/ EP0927 and Arm Gal4;EP0927/EP0927 larvae. Images are taken from muscles 6 and 7 of segment A2. In Arm Gal4;EP0927/EP0927, antiendophilin staining is absent at the motor terminals of the larva shown in the top panel and detected at very low levels in another case shown in the bottom panel. $C$, Anti-CSP and anti-HRP staining at the neuromuscular junction of wild-type Elav Gal4;EP0927/EP0927 and Arm Gal4;EP0927/ EP0927. Note the decrease in size of the boutons in the Arm Gal4; EP0927/EP0927. D, Increase in the number of boutons per synapse in Arm Gal4;EP0927/EP0927 compared with controls. The muscle area is unchanged and is therefore omitted from the calculation. The bouton counts were done on muscles 6 and 7 of segment A2 $(n=13$ for wild type; $n=10$ for Elav Gal4;EP0927/EP0927; and $n=10$ for Arm Gal4;EP0927/ EP0927). Error bars indicate SD. ${ }^{* *} p<0.001$, calculated with one-way ANOVA using the standard Tukey test. Arrow heads point to boutons with endophilin immunoreactivity. $C S$, Caton Special.

third instar larvae showed no defects in locomotion. Intracellular recordings from the larval neuromuscular junction showed that there was an increase in the spontaneous MEJP size and frequency (Fig. 4A). The amplitude of the evoked response showed 
A
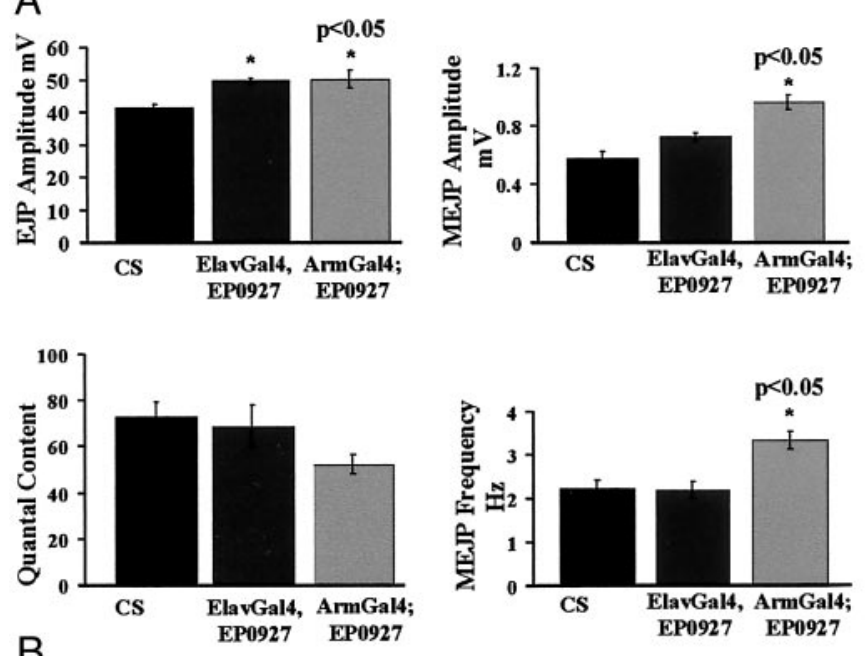

B

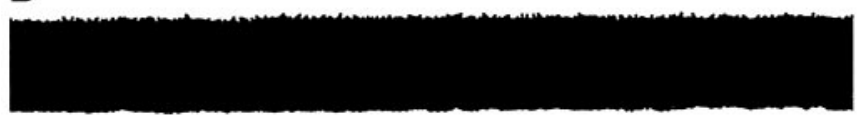

CS

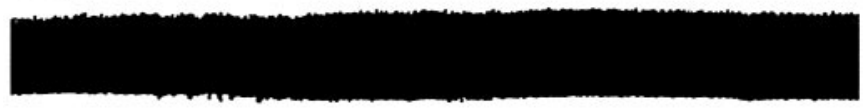

Elav Gal4; EP0927/EP0927

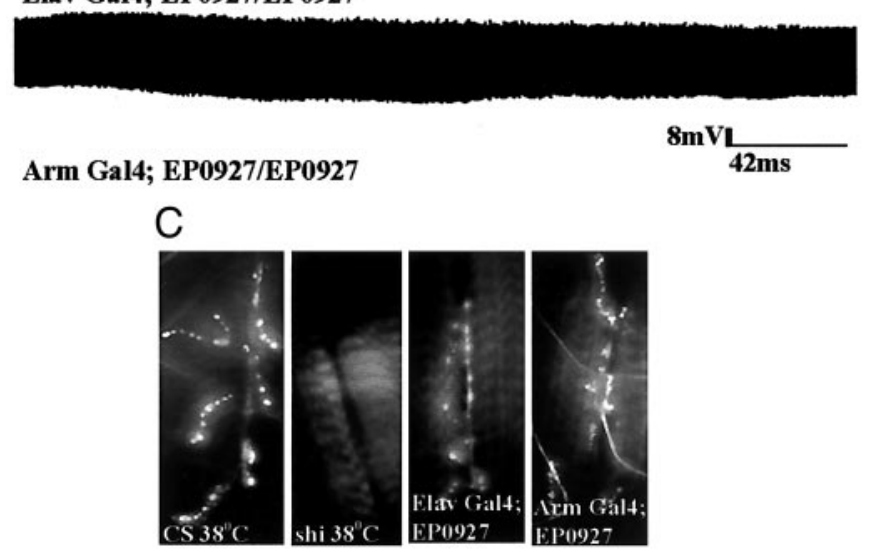

Figure 4. Altered synaptic function at the NMJ of Arm Gal4;EP0927/ EP0927. A, EJP amplitude in wild-type and Arm Gal4;EP0927/EP0927 larvae $\left(n=5\right.$ in each case). Error bars indicate SE; ${ }^{*} p<0.05$. MEJP in wild-type and Arm Gal4;EP0927/EP0927 larvae ( $n=9$ in each case). All recordings are done from muscle 6 of segment A2. Error bars indicate SE; ${ }^{*} p<0.05$. Quantal content calculation is computed by dividing the EJP mean with the MEJP mean. $B$, Synaptic depression protocol applied to wild-type Elav Gal4;EP0927/EP0927 and Arm Gal4;EP0927/EP0927. The larval NMJ was stimulated at $10 \mathrm{~Hz}$ for $5 \min (n=5)$ in each case. The change in amplitude at the start and the end of the stimulus train is not significant. C, FM1-43 uptake in wild-type Elav Gal4;EP0927/EP0927 and Arm Gal4;EP0927/EP0927. The uptake for wild type and $s h i^{t s 2}$ was done at $38^{\circ} \mathrm{C}$ and that in Elav Gal4;EP0927/EP0927 and Arm Gal4;EP0927/ EP0927 was done at room temperature. CS, Canton Special.

a slight increase compared with wild type. Despite the increase in EJP amplitude, we found that the overall quantal content was not significantly affected (Fig. $4 A$ ). We did not observe any signs of synaptic depression consequent to a decrease in endophilin levels at the Drosophila neuromuscular junction (Fig. 4B). This phenotype differs considerably from the total loss of synaptic transmission in the eye observed in the endophilin mutant clones in Figure
1. In Elav Gal4;EP0927/EP0927, although the number of boutons is comparable with wild type, the synaptic physiology is rescued to a level only intermediate between the Arm Gal4;EP0927/ EP0927 and wild type.

\section{FM1-43 uptake is persistent at the} neuromuscular junction

We performed FM1-43 dye-uptake studies at the NMJ of third instar larvae from Arm Gal4;EP0927/EP0927, Elav Gal4 EP0927/ EP0927 shibire ${ }^{t s}$, and wild-type. It is clear that the FM1-43 uptake is not abolished, in contrast to identically treated shi ts larvae at nonpermissive temperatures (Fig. $4 C$ ). There is also no visible difference in uptake between smaller and larger boutons.

\section{DISCUSSION}

In flies with homozygous eye clones for a $\mathrm{P}$ insertion upstream of the endophilin gene (Fig. 1), the on and off transients, when present, peter out and completely vanish very shortly after a train of light pulses is applied. However, the depolarization is undiminished. The transients recrudesce when light flashes are applied after prolonged rest periods in the dark. It is known that in wild-type Drosophila, ERGs are robust and do not show bleach or loss of on-off transients at heightened stimulation. Thus, we observe a novel activity-dependent loss of synaptic transmission in the eye clones that are mutant for endophilin. Such a loss of transients is known to occur in synaptic vesicle-recycling mutants such as shibire after shifting to nonpermissive temperatures (Kelly and Suzuki, 1974). The first few flashes of light in our experiment appear to serve the same purpose as a shift to a nonpermissive temperature in shibire ${ }^{t s}$ mutants. This phenotype is presumably attributable to synapse failure resulting from slowed vesicle recycling. Immunostaining of the NMJ shows considerable coincidence of the staining patterns of dynamin and endophilin (Fig. $2 C$ ). Both of these molecules also show a reticulate pattern; the staining profiles in both cases are exclusive of the glutamate receptor pattern. This points to a role for endophilin in vesicle recycling.

The nervous system-specific staining of anti-endophilin and rescue of the endophilin mutant phenotypes, including lethality using a pan-neuronal Gal4, indicate that it has a predominantly neuronal function. We further assayed an endophilin hypomorph (Arm Gal4;EP0927/EP0927) for phenotypes at the NMJ. Lower expression of endophilin produced a late pupal lethality with few escapers. The third instar larvae were viable and show almost no endophilin immunoreactivity at the NMJ. We observed an apparent change in the synapse size, an increase in the number of boutons, and a decrease in the size of the boutons (Fig. 3). Plasticity of the synapse structure at the Drosophila NMJ is well known. An activity-dependent increase in synapse size has been shown in the case of the hyperactive mutant combination of eag Shaker and by alterations in levels of molecules such as cAMP in dunce (Budnik et al., 1990; Zhong and Wu, 1991; Zhong et al., 1992). A change in synapse size resulting from mutations in components of the synaptic vesicle cycle has been reported in one other case of a lethal mutation of comatose and indirectly in an imbalance of synaptic molecules attributable to dysf unction in the protein degradation machinery in the case of fat facets and highwire (DiAntonio et al., 2001; Sanyal and Krishnan, 2001). The endophilin hypomorph represents one of the few instances of a change in synapse structure via an alteration in the level of a protein involved in the synaptic vesicle cycle. 
This change in the synapse structure seems to alter synapse function in the endophilin hypomorph (Fig. 4). The larger MEJPs observed are possibly reminiscent of other endocytically compromised mutations such as lap (defective in AP180) and stoned (Zhang et al., 1998; Stimson et al., 2001) and are an indication of the importance of endophilin in the precision of vesicle recycling. This is also in agreement with shibire ${ }^{t s 1}$ recovery experiments, in which larger MEJPs are seen during the early stages of recovery from exposure to nonpermissive temperatures (Koenig et al., 1989). It is conceivable that a compromised internalization leads to defective endocytic processing that results in changes in quantal size. It is noteworthy that the quantal content is essentially unaltered in this weak hypomorph; this is reflected in the observation that despite these functional changes, the endophilin hypomorph does not show any ERG defect or depression at the synapse. In our hands, we could not obtain comparable larval stages that were EP0927 homozygous.

Phenotypically, we rate +/+ (wild type) > Elav Gal4;EP0927/ EP0927 > Arm Gal4;EP0927/EP0927 > EP0927/EP0927. Although EP0927/EP0927 is lethal and shows ERG defects, Arm Gal4-rescued flies fail to eclose and have gross changes in bouton structure but show no ERG defects; Elav Gal4-rescued flies are almost normal, show some level of synaptic defects, and produce viable flies that are sluggish. Although vesicle-recycling defects are uncovered in the more severe EP0927 line in the eye clones, the heightened requirement for endophilin activity during development of the nervous system leads to changes in synapse morphology in the less severe hypomorphs.

In conclusion, we have obtained evidence for a neuronal role for endophilin. Our results suggest a role for endophilin in dynamin-mediated synaptic vesicle recycling. We show that a decrease in the levels of the protein leads to significant effects on synapse structure and function. This might in fact be a homeostatic consequence of primarily a vesicle-recycling defect. Finally, the phenotypes obtained suggest the requirement for endophilin and its lipid-modifying activity in the highly regulated form of endocytosis in neurons (Schmidt et al., 1999; Farsad et al., 2001). We surmise that endophilin may play a role in imparting precision to the vesiculation process. The predominantly neuronal role raises the possibility that lysophosphatidic-acid acyl transferase activity is not a strict requirement in all dynamin-mediated endocytosis. In addition, such endocytosis may not require the precision that will be demanded of synaptic vesicle recycling in terms of the components, vesicle size, and time scale. Under conditions in which this is curtailed, the synapse may be able to kick in alternative pathways. Note that if alternative pathways are present in situations in which the regular pathway has been compromised, they could be induced rather than inherent. However, these induced pathways continue to be dynamin-dependent. The FM1-43 results shown are supportive of this contention.

We wish to add that our results are consistent with and further extend reports in two papers (Guichet et al., 2002; Verstreken et al., 2002) that show a requirement of endophilin in dynaminmediated synaptic vesicle recycling.

\section{REFERENCES}

Benzer S (1967) Behavioral mutants of Drosophila isolated by countercurrent distribution. Proc Natl Acad Sci USA 58:1112-1119.

Budnik V, Zhong Y, Wu CF (1990) Morphological plasticity of motor axons in Drosophila mutants with altered excitability. J Neurosci 10:3754-3768.
David C, McPherson PS, Mundigl O, De Camilli P (1996) A role of amphiphysin in synaptic vesicle endocytosis suggested by its binding to dynamin in nerve terminals. Proc Natl Acad Sci USA 93:331-335.

DiAntonio A, Haghighi AP, Portman SL, Lee JD, Amaranto AM, Goodman CS (2001) Ubiquitination-dependent mechanisms regulate synaptic growth and function. Nature 412:449-452.

Estes PS, Roos J, Van der Bliek A, Kelly RB, Krishnan KS, Ramaswami M (1996) Traffic of dynamin within individual Drosophila synaptic boutons relative to compartment-specific markers. J Neurosci 16:5443-5456.

Farsad K, Ringstad N, Takei K, Floyd SR, Rose K, De Camilli P (2001) Generation of high curvature membranes mediated by direct Endophilin bilayer interactions. J Cell Biol 155:193-200.

Gad H, Ringstad H, Löw P, Kjaerulff O, Gustafsson J, Wenk M, Di Paolo G, Nemoto Y, Crum J, Ellisman M, De Camilli P, Shupliakov O, Brodin L (2000) Fission and uncoating of synaptic clathrin-coated vesicles are perturbed by disruption of interactions with the SH3 domain of endophilin. Neuron 27:301-312.

Guichet A, Wucherpfennig T, Dudu V, Etter S, Wilsch-Brauniger M, Hellwig A, Gonzalez-Gaitan M, Huttner WB, Schmidt AA (2002) Essential role of endophilin A in synaptic vesicle budding at the Drosophila neuromuscular junction. EMBO J 21:1661-1672.

Heisenberg M (1971) Separation of receptor and lamina potentials in the electroretinogram of normal and mutant Drosophila. J Exp Biol 55:85-100.

Kelly LE, Suzuki DT (1974) The effects of increased temperature on electroretinograms of temperature-sensitive paralysis mutants of Drosophila melanogaster. Proc Natl Acad Sci USA 71:4906-4909.

Koenig JH, Kosaka T, Ikeda K (1989) The relationship between the number of synaptic vesicles and the amount of transmitter released. J Neurosci 9:1937-1942.

Leventis PA, Chow BM, Stewart BA, Iyengar B, Campos AR, Boulianne GL (2001) Drosophila amphiphysin is a post-synaptic protein required for normal locomotion but not endocytosis. Traffic 2:839-850.

Marks B, Stowell MH, Vallis Y, Mills IG, Gibson A, Hopkins CR, McMahon HT (2001) GTPase activity of dynamin and resulting conformation change are essential for endocytosis. Nature 410:231-235.

Myers EW, Sutton GG, Delcher AL, Dew IM, Fasulo DP, Flanigan MJ, Kravitz SA, Mobarry CM, Reinert KH, Remington KA, Anson EL, Bolanos RA, Chou HH, Jordan CM, Halpern AL, Lonardi S, Beasley EM, Brandon RC, Chen L, Dunn PJ, et al (2000) A whole-genome assembly of Drosophila. Science 287:2196-2204.

Ramaswami M, Krishnan KS, Kelly RB (1994) Intermediates in synaptic vesicle recycling revealed by optical imaging of Drosophila neuromuscular junctions. Neuron 13:363-375.

Razzaq A, Robinson IM, McMahon HT, Skepper JN, Su Y, Zelhof AC, Jackson AP, Gay NJ, O'Kane CJ (2001) Amphiphysin is necessary for organization of the excitation-contraction coupling machinery of muscles, but not for synaptic vesicle endocytosis in Drosophila. Genes Dev 15:2967-2979.

Ringstad N, Nemoto Y, De Camilli P (1997) The SH3p4/Sh3p8/SH3p13 protein family: binding partners for synaptojanin and dynamin via a Grb2-like Src homology 3 domain. Proc Natl Acad Sci USA 94:8569-8574

Ringstad N, Gad H, Löw P, Di Paolo G, Brodin L, Shupliakov O, De Camilli P (1999) Endophilin/SH3p4 is required for the transition from early to late stages in clathrin-mediated synaptic vesicle endocytosis. Neuron 24:143-154.

Roos J, Kelly RB (1999) The endocytic machinery in nerve terminals surrounds sites of exocytosis. Curr Biol 9:1411-1414.

Rorth P (1996) A modular misexpression screen in Drosophila detecting tissue-specific phenotypes. Proc Natl Acad Sci USA 93:12418-12422.

Sanyal S, Krishnan KS (2001) Lethal comatose mutation in Drosophila reveals possible role for NSF in neurogenesis. NeuroReport 12:1363-1366.

Schmidt A, Wolde M, Thiele C, Fest W, Kartzin H, Podtelejnikov AV, Witke W, Huttner WB, Soling HD (1999) Endophilin I mediates synaptic vesicle formation by transfer of arachidonate to lysophosphatidic acid. Nature 401:133-141.

Shupliakov O, Low P, Grabs D, Gad H, Chen H, David C, Takei K, De Camilli P, Brodin L (1997) Synaptic vesicle endocytosis impaired by disruption of dynamin-SH3 domain interactions. Science 276:259-263.

Simpson F, Hussain NK, Qualmann B, Kelly RB, Kay BK, McPherson PS, Schmid SL (1999) SH3-domain-containing proteins function at distinct steps in clathrin-coated vesicle formation. Nat Cell Biol 1:119-124.

Stimson DT, Estes PS, Rao S, Krishnan KS, Kelly LE, Ramaswami M (2001) Drosophila stoned proteins regulate the rate and fidelity of synaptic vesicle internalization. J Neurosci 21:3034-3044.

Stowell MH, Marks B, Wigge P, McMahon HT (1999) Nucleotidedependent conformational changes in Dynamin: evidence for a mechanochemical molecular spring. Nat Cell Biol 1:27-32.

Stowers RS, Schwarz TL (1999) A genetic method for generating Dro- 
sophila eyes composed exclusively of mitotic clones of a single genotype. Genetics 152:1631-1639.

Takei K, Haucke V, Slepnev V, Farsad K, Salazar M, Chen H, De Camilli P (1998) Generation of coated intermediates of clathrin-mediated endocytosis on protein-free liposomes. Cell 1998 94:131-141.

Takei K, Slepnev VI, Haucke V, De Camilli P (1999) Functional partnership between amphiphysin and dynamin in clathrin-mediated endocytosis. Nat Cell Biol 1:33-39.

Verstreken P, Kjaerulff O, Lloyd TE, Atkinson R, Zhou Y, Meinertzhagen IA, Bellen HJ (2002) Endophilin mutations block clathrinmediated endocytosis but not neurotransmitter release. Cell 109:101-112.
Zelhof AC, Bao H, Hardy RW, Razzaq A, Zhang B, Doe CQ (2001) Drosophila amphiphysin is implicated in protein localization and membrane morphogenesis but not in synaptic vesicle endocytosis. Development 128:5005-5015.

Zhang B, Koh YH, Beckstead RB, Budnik V, Ganetzky B, Bellen HJ (1998) Synaptic vesicle size and number are regulated by a clathrin adaptor protein required for endocytosis. Neuron 21:1465-1475.

Zhong Y, Wu CF (1991) Altered synaptic plasticity in Drosophila memory mutants with a defective cyclic AMP cascade. Science 251:198-201.

Zhong Y, Budnik V, Wu CF (1992) Synaptic plasticity in Drosophila memory and hyperexcitable mutants: role of cAMP cascade. J Neurosci 12:644-651. 